\title{
Rico and the jets: Direct numerical simulations of turbulent liquid jets
}

\author{
C. R. Constante-Amores $\odot,{ }^{1, *}$ L. Kahouadji $\odot,{ }^{1}$ A. Batchvarov, ${ }^{1}$ S. Shin, ${ }^{2}$ J. Chergui $\odot,{ }^{3}$ \\ D. Juric, ${ }^{3}$ and O. K. Matar $\left(^{1}\right.$ \\ ${ }^{1}$ Department of Chemical Engineering, Imperial College London, South Kensington Campus, \\ London SW7 2AZ, United Kingdom \\ ${ }^{2}$ Department of Mechanical and System Design Engineering, Hongik University, \\ Seoul 121-791, Republic of Korea \\ ${ }^{3}$ Laboratoire d'Informatique pour la Mécanique et les Sciences de l'Ingénieur (LIMSI), \\ Centre National de la Recherche Scientifique (CNRS), Université Paris Saclay, Bât. 507, \\ Rue du Belvédère, Campus Universitaire, 91405 Orsay, France
}

(Received 24 July 2020; published 12 November 2020)

\begin{abstract}
This paper is associated with a poster winner of a 2019 American Physical Society's Division of Fluid Dynamics (DFD) Milton van Dyke Award for work presented at the DFD Gallery of Fluid Motion. The original poster is available online at the Gallery of Fluid Motion, https://doi.org/10.1103/APS.DFD.2019.GFM.P0020.
\end{abstract}

DOI: 10.1103/PhysRevFluids.5.110501

The breakup of an interface into a cascade of droplets and their subsequent coalescence is a generic problem of central importance to a large number of industrial settings. Examples of these applications include the atomization of propellants in engines, the formation of droplets in injectors, mixers, and separators, and the generation of droplets in multiphase flow regime transitions [1-3]. In all of these situations, it is important to predict the evolving droplet size distribution that results from a competition between breakup and coalescence, which are influenced by a range of multiscale physics; this includes the interaction of turbulence with interfaces, capillarity, viscosity, and gravity. Therefore, it is unsurprising that the breakup of liquid jets during injection (i.e., atomization) has received great scientific interest [4-8].

The transient dynamics of turbulent liquid/liquid systems have not received the attention they deserve in the literature. Temporal instabilities and the resulting spatiotemporal interfacial structures are predicted by solving the full three-dimensional two-phase Navier-Stokes system in the context of a hybrid front-tracking/level-set method [9-11]. We consider a cylindrical nozzle with diameter $D=$ $4 \mathrm{~mm}$ injecting water with density $\rho_{w}$ and dynamic viscosity $\mu_{w}$. This water jet enters progressively into the computational domain of size $20 D \times 4 D \times 4 D$, initially filled with a stagnant viscous silicone oil of density $\rho_{s o}$ and viscosity $\mu_{s o}$. The surface tension is taken to be that of oil and water (e.g., $\sigma=35.1 \mathrm{mN} / \mathrm{s}$ ) [12]. The Reynolds number is defined as $\operatorname{Re}=\rho_{w} U_{\text {jet }} D / \mu_{w}$ and fixed to the value of $\operatorname{Re}=6530$. The domain has been divided into $48 \times 6 \times 6$ subdomains where each subdomain contains a Cartesian structured grid of $64^{3}$ cells, accounting for a global structured mesh grid of $3072 \times 384 \times 384$. This mesh is sufficiently large to resolve all relevant turbulent length scales and interfacial singularities (e.g., pinch-off and coalescence).

\footnotetext{
*crc15@imperial.ac.uk
}

Published by the American Physical Society under the terms of the Creative Commons Attribution 4.0 International license. Further distribution of this work must maintain attribution to the author(s) and the published article's title, journal citation, and DOI. 


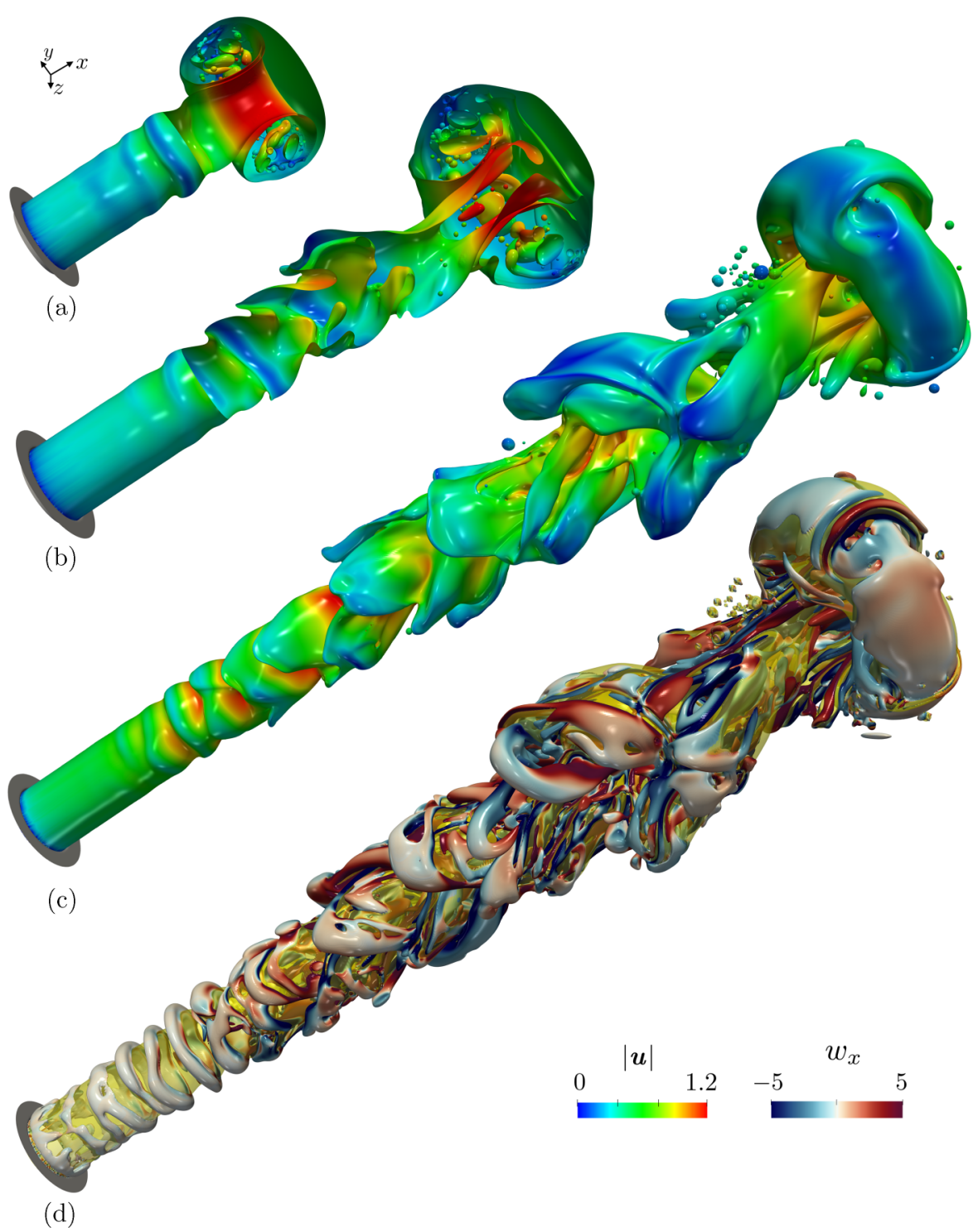

FIG. 1. Spatiotemporal evolution of the interface in the injection of a water jet into a stagnant viscous silicone oil at $t=(7.25,12.05,28.97)$ corresponding to (a), (b), and (c), respectively, with $\operatorname{Re}=6530$. (d) Illustration of the coherent vortical structures through the $Q$ criterion close to the free surface (in yellow) at $t=28.97$. The vortical structures have been colored by the value of the vorticity in the streamwise direction. In the vorticity representation, blue and red represent vortical structures with counterclockwise and clockwise rotation, respectively. All variables are dimensionless quantities.

We use a solver for massively parallel simulations of fully three-dimensional multiphase flows [10], able to run on a variety of computer architectures, wholly written in FORTRAN 2008 and adopting an algebraic domain decomposition strategy for parallelization with MPI. The fluid interface solver is based on a parallel implementation of the Level Contour Reconstruction Method (LCRM), which is an adaptation of our high-fidelity hybrid front-tracking/level-set method, able to handle highly deforming interfaces with complex topology changes [13-15]. This code uses parallel GMRES and multigrid iterative solvers suited to solve the linear systems arising from the implicit 
solution of the fluid velocities and pressure. More details on the numerical techniques can be found in Shin et al. [10,11].

The spatiotemporal evolution of the interfacial dynamics is shown in Fig. 1. At early injection times, large capillary pressure is generated near the leading edge, due to local interfacial curvature, leading to a radially-driven flow. This capillary-induced flow together with the viscous resistance from the stagnant phase yields the formation of a leading-top mushroom-like structure [see Fig. 1(a)]. This structure covers an internal interfacial toroidal sheet whose thickness reduces over time to generate the formation of holes, which expand radially to form ligaments and eventually entrapped droplets. As time evolves, the free surface behind the leading structure adopts the shape of a "cylinder" which undergoes a Kelvin-Helmholtz instability $(\mathrm{KH})$ to form the initial corrugations observed to occur on the free surface. The $\mathrm{KH}$ instabilities on the free surface are triggered by the parallel motion of fluids at different velocities and are amplified by the pulsatile injection.

The interfacial dynamics of the jet can be explained by coupling the vorticity $\boldsymbol{\omega}=\boldsymbol{\nabla} \times \mathbf{u}$ with the interfacial location. During the early stages and close to the injection point, the streamwise vorticity field $\omega_{x}$ is characterized by values which are two orders of magnitude smaller than the azimuthal vorticity $\omega_{\theta}$. As the flow evolves downstream, $\omega_{x}$ becomes comparable in magnitude with $\omega_{\theta}$, leading to the deformation of axisymmetric $\mathrm{KH}$ vortex rings in the streamwise direction adopting a new hairpin shape. These hairpin vortices trigger the formation of interfacial lobes which are stretched downstream (from outer hairpin vortices) and upstream (from inner hairpin vortices) to eventually obtain a hairpin shape [see Figs. 1(b) and 1(c)]. We have used the $Q$ criterion to visualize the three-dimensional nature of the vortical structure [shown in Fig. 1(d)] [16]. The topological shape of the vortex resembles the instantaneous hairpin-like vortical structures reported in experiments and numerical simulations of $[17,18]$. Outer hairpin vortices are observed clearly, whereas the inner hairpin vortices are localized underneath the interface. Additionally, a vortex-cap covers the leading mushroom structure.

This work is supported by the Engineering \& Physical Sciences Research Council of the United Kingdom, through a studentship for R.C.A. in the Centre for Doctoral Training on Theory and Simulation of Materials at Imperial College London funded by the EPSRC (EP/L015579/1; award reference 1808927) and through the EPSRC MEMPHIS (EP/K003976/1) and PREMIERE (EP/T000414/1) Programme Grants. O.K.M. also acknowledges funding from PETRONAS and the Royal Academy of Engineering for a Research Chair in Multiphase Fluid Dynamics. We also acknowledge HPC facilities provided by the Research Computing Service (RCS) of Imperial College London for the computing time. D.J. and J.C. acknowledge support through computing time at the Institut du Developpement et des Ressources en Informatique Scientifique (IDRIS) of the Centre National de la Recherche Scientifique (CNRS), coordinated by GENCI (Grand Equipement National de Calcul Intensif) Grant No. 2020 A0082B06721. The numerical simulations were performed with code BLUE [10], and the visualizations have been generated using ParaView.

[1] J. Eggers, Nonlinear dynamics and breakup of free-surface flows, Rev. Mod. Phys. 69, 865 (1997).

[2] J. Eggers and E. Villermaux, Physics of liquid jets, Rep. Prog. Phys. 71, 036601 (2008).

[3] S. P. Lin and R. D. Reitz, Drop and spray formation from a liquid jet, Annu. Rev. Fluid Mech. 30, 85 (1998).

[4] O. Desjardins and H. Pitsch, Detailed numerical investigation of turbulent atomization of liquid jets, Atomiz. Sprays 20, 311 (2010).

[5] E. Ibarra, F. Shaffer, and O. Savaş, On the near-field interfaces of homogeneous and immiscible round turbulent jets, J. Fluid Mech. 889, A4 (2020).

[6] D. Jarrahbashi, W. A. Sirignano, P. P. Popov, and F. Hussain, Early spray development at high gas density: Hole, ligament and bridge formations, J. Fluid Mech. 792, 186 (2016). 
[7] J. C. Lasheras and E. J. Hopfinger, Liquid jet instability and atomization in a coaxial gas stream, Annu. Rev. Fluid Mech. 32, 275 (2000).

[8] P. Marmottant and E. Villermaux, On spray formation, J. Fluid Mech. 498, 73 (2004).

[9] S. Shin and D. Juric, Modeling three-dimensional multiphase flow using a level contour reconstruction method for front tracking without connectivity, J. Comput. Phys. 180, 427 (2002).

[10] S. Shin, J. Chergui, and D. Juric, A solver for massively parallel direct numerical simulation of threedimensional multiphase flows, J. Mech. Sci. Tech. 31, 1739 (2017).

[11] S. Shin, J. Chergui, D. Juric, L. Kahouadji, O. K. Matar, and R. V. Craster, An interface-tracking technique for multiphase flow with soluble surfactant, J. Comput. Phys. 359, 409 (2018).

[12] R. Ibarra, Horizontal and low-inclination oil-water flow investigations using laser-based diagnostic techniques, Ph.D. thesis, Imperial College London (2017).

[13] A. Batchvarov, L. Kahouadji, C. R. Constante-Amores, G. F. N. Gonõalves, S. Shin, J. Chergui, D. Juric, and O. K. Matar, Three-dimensional dynamics of falling films in the presence of insoluble surfactants, J. Fluid Mech. (2020), arXiv:2005.12669.

[14] C. R. Constante-Amores, L. Kahouadji, A. Batchvarov, S. Shin, J. Chergui, D. Juric, and O. K. Matar, Dynamics of retracting surfactant-laden ligaments at intermediate Ohnesorge number, Phys. Rev. Fluids 5, 084007 (2020).

[15] C. R. Constante-Amores, L. Kahouadji, A. Batchvarov, S. Shin, J. Chergui, D. Juric, and O. K. Matar, Dynamics of a surfactant-laden bubble bursting through an interface, arXiv:2005.04472.

[16] J. C. R. Hunt, A. A. Wray, and P. Moin, Eddies, stream, and convergence zones in turbulent flows, Center for Turbulent Research Report CTR-S88 (1988).

[17] A. Zandian, W. A. Sirignano, and F. Hussain, Understanding liquid-jet atomization cascades via vortex dynamics, J. Fluid Mech. 843, 293 (2018).

[18] J. Zhou, R. J. Adrian, S. Balachandar, and T. M. Kendall, Mechanisms for generating coherent packets of hairpin vortices in channel flow, J. Fluid Mech. 387, 353 (1999). 